Original Article

\title{
Preoperative physical performance predictors of self-reported physical function and quality of life in patients scheduled for total knee arthroplasty
}

\author{
Chul Woong Hyun, MD²), Bo Ryun Kim, MD, PhD²), Eun Young Han, MD, PhD $^{2}$, \\ SANG Rim KIm, MD, PhD ${ }^{3}$ \\ 1) Department of Rehabilitation Medicine, Lohas Gwangmyeong Convalescent Hospital, \\ Republic of Korea \\ 2) Department of Rehabilitation Medicine, Jeju National University Hospital, Jeju National University \\ School of Medicine: Aran 13 gil 15, Jeju-si, Jeju 690-767, Republic of Korea \\ 3) Department of Orthopedic Surgery, Jeju National University Hospital, Jeju National University \\ College of Medicine, Republic of Korea
}

\begin{abstract}
Purpose] To determine the preoperative self-reported and performance-based physical function of patients with end-stage knee osteoarthritis who awaited total knee arthroplasty. The preoperative physical performance factors that predicted self-reported physical function and quality of life were also identified. [Subjects and Methods] All adults with end-stage knee osteoarthritis awaiting surgery were enrolled. Before surgery, self-reported disease-specific physical function and self-reported pain were measured using the Western Ontario McMaster Universities Osteoarthritis Index, self-reported quality of life was measured using the EuroQOL five dimensions questionnaire, and physical performance tests were performed, the 6 minute walk test, the timed up-and-go test, instrumental gait analysis, and measurement of isometric knee flexor and extensor strength of the surgical and nonsurgical knees. [Results] In total, 55 adults (49 females; $73.3 \pm 6.1$ years) were included. This study showed that several preoperative self-reported and physical performance factors were predictive of self-reported physical function and quality of life. [Conclusion] In patients with end-stage knee osteoarthritis, preoperative pain and dynamic balance ability were the most powerful predictors of self-reported physical function. Preoperative pain and exercise tolerance were the most powerful predictors of quality of life. Preoperative rehabilitation strategies that focus on dynamic balance, aerobic, and resistance exercises may improve surgical outcomes.

Key words: Total knee arthroplasty, Function, Quality of life
\end{abstract}

(This article was submitted Jun. 20, 2016, and was accepted Jul. 29, 2016)

\section{INTRODUCTION}

End-stage knee osteoarthritis (OA) is one of the most common debilitating diseases and is a leading cause of pain, stiffness, edema, disability, and functional limitation in older adults ${ }^{1-3)}$. Several studies have reported that when patients with end-stage knee OA are asked to complete a self-reported questionnaire before surgery, they tend to have markedly lower scores than people without knee OA. In particular, the patients have poor scores for selected functional items ${ }^{4-6)}$. They are also more likely to have impaired standing balance ${ }^{7}$, progressive loss of function that causes increasing dependency while walking ${ }^{1)}$, and decreased knee extensor strength ${ }^{8)}$. Total knee arthroplasty (TKA) is the treatment of choice for end-stage knee OA as it is the most effective surgical procedure for this condition ${ }^{4,9}$.

However, nearly all of these studies were performed with Western patients, which suggest that their observations may

*Corresponding author. Bo Ryun Kim (E-mail: brkim08@gmail.com)

(C2016 The Society of Physical Therapy Science. Published by IPEC Inc.

This is an open-access article distributed under the terms of the Creative Commons Attribution Non-Commercial No Derivatives (by-nc-nd) License $<$ http://creativecommons.org/licenses/by-nc-nd/4.0/>. 
not be generalizable to patients with knee OA in other ethnicities or regions. This may be particularly true for South Korea because most South Korean patients with knee OA tend to undergo TKA at a more advanced stage than patients in Western countries. This may reflect the fact that the South Korean population is relatively sedentary and thus less affected by the pain and disability that accompanies knee OA. Therefore, it is important to determine the baseline preoperative physical function of patients with end-stage knee OA in South Korea. At present, such studies are rare.

As indicated above, physical function can be measured by asking the patient to complete a self-reported questionnaire. Indeed, in patients who are scheduled to undergo TKA, orthopedic surgeons mainly utilize questionnaires such as the Western Ontario McMaster Universities Osteoarthritis Index (WOMAC) to evaluate preoperative functional capability and to predict postoperative functional improvement ${ }^{10,11)}$ : several recent studies have shown that preoperative self-report questionnaire scores consistently predict postoperative questionnaire scores ${ }^{4-6}$. Another way to measure physical function is to perform physical function tests such as the 6 minute walk test (6MWT). However, studies that have used both questionnaires and physical function tests have found that the self-perceived physical function of individuals often differs substantially from their actual functional capability ${ }^{12,13}$. In particular, several studies have shown that the functional test scores of patients with knee OA correlate only modestly with their self-reported questionnaire scores ${ }^{14,15)}$. Notably, recent studies have shown that functional test scores before and after TKA correlate strongly. Thus, to best predict TKA outcomes, it may be necessary to apply both self-reported questionnaires of functional status and physical function tests.

Some studies have shown that in preoperative knee OA, several physical function test variables are predictive of selfreported functional status ${ }^{16-18}$. This information is important because it can indicate which preoperative rehabilitative interventions would best improve the outcomes of TKA for knee OA. However, at present, the preoperative physical function test variables that best predict self-reported functional ability in South Koreans with knee OA are unknown. To address these questions, this prospective cross-sectional study was performed. Its aim was to delineate the preoperative self-reported and performance-based physical function of South Korean patients with end-stage knee OA who were awaiting a TKA. Moreover, to determine the preoperative rehabilitation strategies that could improve the functional capacity of these patients, the study identified the preoperative physical performance factors that predicted self-reported physical function and quality of life.

\section{SUBJECTS AND METHODS}

All consecutive older adults with end-stage primary OA of the knee who were waiting for a first TKA in a tertiary referral hospital were enrolled prospectively between October 2013 and July 2014. All enrolled patients were required to be ambulatory with or without a walking aid and to have a radiological score of III-IV, as determined using the Kellgren-Lawrence method.

Each subject received information about the study and gave written informed consent form to participate. And our local Ethics Committee approved the study protocol.

All 55 patients completed the following assessments before surgery. Self-reported disease-specific physical function and self-reported pain were measured using the WOMAC. Self-reported quality of life was measured using the EuroQOL five dimensions (EQ-5D) questionnaire. Physical performance tests included the 6MWT, the timed up-and-go (TUG) test, instrumental gait analysis for spatiotemporal variables, and measurement of the isometric knee extensor and flexor strength of the surgical and nonsurgical knees.

Western Ontario McMaster Universities Osteoarthritis Index (WOMAC): WOMAC is a multidimensional questionnaire that asks questions about pain, stiffness, and physical function. It was used previously to measure self-reported disability in patients with knee OA 20. There are 5 pain variables, 2 stiffness variables, and 17 physical function variables. Each of the 24 WOMAC variables is scored using the Likert scale ( 0 , none; 1 , slight; 2 , moderate; 3 , very; and 4, extremely), as recommended by the Outcome Measures in Rheumatology Clinical Trials (OMERACT). Thus, the Likert scale is employed to determine the degree of pain, stiffness, and difficulty experienced in performing each of 17 activities in the preceding 48 hours. Higher scores indicate greater levels of pain, stiffness, and difficulty ${ }^{19)}$. The scores of pain, stiffness, and physical function variables are added to yield the WOMAC-Pain (range 0-20), WOMAC-Stiffness (range 0-8), and WOMAC-Function (range 0-68) subscores.

EuroQOL five dimensions (EQ-5D) questionnaire: Self-reported quality of life was measured using the EQ-5D questionnaire. The EQ-5D Index is widely used to measure general health status. The instrument comprises a questionnaire with five dimensions: mobility, self-care, usual activities, pain/discomfort, and anxiety/depression. Each dimension is represented by one question with three severity levels (no problems, some or moderate problems, and extreme problems) 21. Scores were transformed using utility weights derived from the general Korean population 21 and ranged from -1 to 1 . Higher scores indicated better overall health status. The formula producing the EQ-5D Index has been presented by Kim et $\mathrm{al}^{20)}$.

Timed up-and-go (TUG) test: The TUG measures dynamic balance while the individual performs physical tasks that are common in everyday life. The TUG tool has been shown to have acceptable concurrent validity in terms of predicting dynamic balance ${ }^{21}$. The TUG test is performed by first asking the patient to wait by sitting with their back against a chair (seat height, $44 \mathrm{~cm}$; depth, $45 \mathrm{~cm}$; width, $49 \mathrm{~cm}$; and arm rest height, $64 \mathrm{~cm}$ ) that has been placed at the end of a marked $3 \mathrm{~m}$ walkway ${ }^{22}$. The patient is then told that when the instructor says "go," the patient should stand up, walk at a comfortable speed past the 3 m mark, turn around, walk back, and sit down in the chair. The time is measured in seconds. Six minute walk 
test (6MWT): The 6MWT is a commonly used standardized measure of exercise tolerance and functional walking capacity in people with compromised mobility. All patients were instructed to walk along a $50 \mathrm{~m}$ track without disturbances. They could use their customary assisting devices during the test but were requested to walk without support when possible.

Gait analysis: Spatiotemporal variables of gait were assessed using a validated 24 wireless inertial sensing device (GSensor1, BTS Bioengineering S.p.A., Italy). The device was attached to the patient's waist using a semi-elastic belt. The belt covers the L4-L5 intervertebral space so that the acceleration values for the three anatomical axes (anteroposterior, mediolateral, and vertical) can be measured. Patients were asked to walk along an $8 \mathrm{~m}$ pathway at a self-selected speed as naturally as possible. The collected data were transmitted via Bluetooth to a personal computer and processed using dedicated software (BTS G-Walk $^{\circledR}$ ). The gait variables that were measured were gait speed, cadence, stride length, gait cycle duration, stance phase duration, swing phase duration, double support duration, and single support duration ${ }^{23}$.

The maximal isometric strength of the bilateral knee extensors and flexors was measured using the isokinetic dynamometer CSMI ${ }^{\circledR}$ (Humac Norm, Stoughton, MA, USA). Patients were seated on a specially designed chair with hip angles of approximately $85^{\circ 24}$. The distal shin pad of the dynamometer was attached $2-3 \mathrm{~cm}$ proximal to the lateral malleolus using a strap. To minimize inappropriate trunk movements during thigh muscle contractions, straps were applied across the chest, pelvis, and mid-thigh. The alignment between the dynamometer rotational axis and the knee joint rotation axis (lateral femoral epicondyle) was adjusted at the beginning of each trial. Gravity effect torque was recorded on each subject. This was used to correct torque measurements during all tests. Patients were asked to grasp sidebars during the testing procedure. The length of the moment arm measured from the lateral femoral epicondyle to the center of the force transducer at the shin was kept constant. Data were obtained from digitized signals. After a structured warm-up, with the knee joint fixed at an angle of $60^{\circ}$ of flexion for maximal isometric force generation ${ }^{25)}$, patients were asked to perform maximal voluntary contractions until the torque did not increase further by more than $5 \%$ during the three successive attempts. Knee flexion and extension were performed as discrete movements in a single direction (i.e. non-reciprocal). Each contraction lasted 4 to 5 seconds. The contractions were separated by 2 minutes of rest. During each attempt, the physical therapist loudly encouraged the patients to achieve their maximal performance. After a 5 minute rest, the same procedure was performed with another lower limb. The variables that were analyzed were peak torque (PT) extensor of the surgical and nonsurgical knees, PT flexor of the surgical and nonsurgical knees, the ratio of hamstrings and quadriceps (H/Q ratio) of the surgical and nonsurgical knees, and the difference between the surgical and nonsurgical knees in terms of extensor and flexor strength (the latter variables were expressed as percentages of the nonsurgical knee performance).

All statistical analyses were performed using SPSS for Windows version 20.0 (SPSS V 20.0 K, SPSS Inc., Chicago, IL, USA). All variables were subjected to descriptive statistics. Pearson's correlation analysis was used to assess the relationships between self-reported physical function or quality of life and physical performance test values. Multivariate linear regression analysis using a backward selection linear regression model was employed to determine which physical performance variables best predicted self-reported physical function and quality of life. $\mathrm{P}<0.05$ were considered to indicate statistical significance.

\section{RESULTS}

In total, 55 consecutive adults with end-stage knee OA who were waiting to undergo TKA met the eligibility criteria and were enrolled in this study. The preoperative demographic and disease-related characteristics of the patients are presented in Table 1. There were 49 females and six men, and the average age was $73.3 \pm 6.12$ (range, 56-86) years. The average body mass index was $25.5 \pm 3.15 \mathrm{~kg} / \mathrm{m}^{2}$. Of the 55 patients, $43(78.2 \%)$ had Kellgren-Lawrence grade IV and $19(34.5 \%)$ had osteoporosis.

The average preoperative physical function, quality of life, and physical test performances of the patients are presented in Table 2. The average WOMAC-Pain, WOMAC-Stiffness, WOMAC-Function, and WOMAC-Total scores were 10.5 \pm 3.1 , $3.0 \pm 1.7,29.3 \pm 10.7$, and $43.1 \pm 13.4$, respectively. The average EQ-5D score was $0.5 \pm 0.3$. The average $6 \mathrm{MWD}$ and TUG values were $296.2 \pm 96.7 \mathrm{~m}$ and $13.3 \pm 7.3$ seconds, respectively. Gait analysis showed that the average gait speed, cadence, stride length, and gait cycle duration were $0.8 \pm 0.2 \mathrm{~m} / \mathrm{sec}, 95.9 \pm 22.0 \mathrm{steps} /$ minute, $93.1 \pm 16.5 \mathrm{~cm}$, and $1.2 \pm 0.2 \mathrm{~seconds}$, respectively. In terms of isometric knee muscle strength, the average PT extensor values of the surgical and nonsurgical knees were $42.1 \pm 19.7$ and $54.6 \pm 21.8 \mathrm{Nm}$, respectively, while the average PT flexor values of the surgical and nonsurgical knees were $32.5 \pm 10.7$ and $37.5 \pm 12.6 \mathrm{Nm}$, respectively.

The WOMAC-Function score correlated positively with the WOMAC-Pain score $(r=0.62, p<0.001)$ and TUG $(r=0.46$, $\mathrm{p}<0.001)$, and negatively with 6MWT $(\mathrm{r}=-0.46, \mathrm{p}<0.001)$ and the PT extensor and flexor values of the nonsurgical knee $(\mathrm{r}=-0.27, \mathrm{p}=0.04$ and $\mathrm{r}=-0.32, \mathrm{p}=0.02$, respectively) (Table 3$)$. After adjustment for demographics and anthropometric variables, linear regression analysis showed that the WOMAC-Pain score $(\beta=0.55, \mathrm{p}<0.001)$ and TUG $(\beta=0.34, \mathrm{p}=0.002)$ were significant independent predictors of the preoperative WOMAC-Function score $(\mathrm{R} 2=0.49)$ (Table 4$)$.

The EQ-5D score correlated positively with 6MWT $(\mathrm{r}=0.46, \mathrm{p}<0.001)$ and negatively with the WOMAC-Pain score $(\mathrm{r}=-0.60, \mathrm{p}<0.001)$ and TUG $(\mathrm{r}=-0.38, \mathrm{p}=0.004)$ (Table 3$)$. After adjustment for demographics and anthropometric variables, the linear regression analysis showed that the WOMAC-Pain score $(\beta=-0.51, \mathrm{p}<0.001)$ and $6 \mathrm{MWT}(\beta=0.30, \mathrm{p}=0.008)$ were significant independent predictors of the EQ-5D score $\left(\mathrm{R}^{2}=0.45\right)$ (Table 4$)$. 
Table 1. Demographic and disease-related characteristics of the patients $(\mathrm{N}=55)$

\begin{tabular}{lc}
\hline Variables & Values \\
\hline Age (years) & $73.3 \pm 6.1^{*}$ \\
Gender, males/females & $6 / 49$ \\
Height $(\mathrm{cm})$ & $153.7 \pm 7.9^{*}$ \\
Weight $(\mathrm{kg})$ & $60.8 \pm 8.8^{*}$ \\
BMI $\left(\mathrm{kg} / \mathrm{m}^{2}\right)$ & $25.5 \pm 3.2^{*}$ \\
K-L grades & \\
Grade 3 & 12 \\
Grade 4 & 43 \\
Lesion side & \\
Left & 20 \\
Right & 21 \\
Both & 14 \\
Comorbidities & \\
Cardiovascular disease & 9 \\
Diabetes mellitus & 7 \\
Hypertension & 9 \\
Osteoporosis & 19 \\
\hline
\end{tabular}

*Values represent mean \pm standard deviation of cases. BMI: body mass index, K-L: Kellgren-Lawrence
Table 2. The preoperative evaluation of self-reported physical function and quality of life, and physical performance

\begin{tabular}{lc}
\hline Variables & Values \\
\hline WOMAC-Pain & $10.5 \pm 3.1^{*}$ \\
WOMAC-Stiffness & $3.0 \pm 1.7^{*}$ \\
WOMAC-Function & $29.3 \pm 10.7^{*}$ \\
WOMAC-Total & $43.1 \pm 13.4^{*}$ \\
EQ-5D & $0.5 \pm 0.3^{*}$ \\
6MWT (m) & $296.2 \pm 96.7^{*}$ \\
TUG (sec) & $13.3 \pm 7.3^{*}$ \\
Gait analysis & \\
Gait speed (m/sec) & $0.8 \pm 0.2^{*}$ \\
Cadence (steps/min) & $95.9 \pm 22.0^{*}$ \\
Stride length (cm) & $93.1 \pm 16.5^{*}$ \\
Gait cycle duration (sec) & $1.2 \pm 0.2^{*}$ \\
Stance phase duration (\% of gait cycle) & $67.0 \pm 3.3^{*}$ \\
Swing phase duration (\% of gait cycle) & $33.0 \pm 3.3^{*}$ \\
Double support duration (\% of gait cycle) & $34.2 \pm 8.2^{*}$ \\
Single support duration (\% of gait cycle) & $32.9 \pm 4.3^{*}$ \\
Isometric strength test & \\
PT extensor of surgical knee (Nm) & $42.1 \pm 19.7^{*}$ \\
PT extensor of non-surgical knee (Nm) & $54.6 \pm 21.8^{*}$ \\
PT flexor of surgical knee (Nm) & $32.5 \pm 10.7^{*}$ \\
PT flexor of non-surgical knee (Nm) & $37.5 \pm 12.6^{*}$ \\
H/Q ratio of surgical knee (\%) & $76.0 \pm 48.6^{*}$ \\
H/Q ratio of non-surgical knee (\%) & $65.0 \pm 34.4^{*}$ \\
Deficit of extensor (\%) & $22.8 \pm 16.7^{*}$ \\
Deficit of flexor (\%) & $12.3 \pm 17.6^{*}$ \\
\hline
\end{tabular}

*Values represent mean \pm standard deviation.

WOMAC: Western Ontario McMaster Universities Osteoarthritis Index, EQ-5D: EuroQOL five dimensions, 6MWT: 6-minute walk test, TUG: timed up and go, PT: peak torque, H/Q: Hamstring/Quadriceps

\section{DISCUSSION}

This study of patients with end-stage knee OA who were awaiting a TKA showed that several preoperative self-reported and physical performance factors were predictive of self-reported physical function and quality of life.

The first key observation of our study was that our patients had a worse self-reported physical function and physical performance than similar patients living in Western countries. Specifically, our patients tended to have higher WOMAC-Pain and WOMAC-Function scores than Western patients, which indicates that our patients reported having more knee pain and disability ${ }^{26}$. Moreover, our patients took twice as long to perform the TUG test and only managed to walk $70 \%$ of the 6MWT distance that was walked by Western patients on average ${ }^{18}$. Our patients also tended to have more knee weakness than Western patients ${ }^{27}$. These differences may be the result of the difference between our cohort and the Western cohorts in terms of age: our patients were older than the Western patients. In turn, this may reflect the tendency of South Korean patients with knee OA to delay undergoing TKA as long as possible. It may also reflect the different lifestyles of South Koreans and Westerners. In particular, South Koreans tend to be more sedentary, which may mean that they are less troubled by their knee pain and disability during everyday life than Westerners. These differences between South Koreans and Westerners illustrate the importance of determining the preoperative self-reported quality of life and disease-specific physical function and pain of patients with end-stage knee OA in non-Western countries.

This information is also important because it allows the identification of the physical test factors that correlate with self-reported physical function and quality of life in specific populations. This in turn will help to identify the pre- and postoperative rehabilitation programs that would best improve the functional capacity of patients with OA who are scheduled to undergo TKA. The second key observation of our study was that self-reported pain, dynamic balance, and exercise tolerance correlated significantly with self-reported physical function and/or quality of life in our patients. Similarly, some studies have 
Table 3. Correlation among preoperative self-reported physical function and quality of life and physical performance

\begin{tabular}{lcc}
\hline & $\begin{array}{c}\text { Correlation coefficients (r) } \\
\text { Preoperative }\end{array}$ & $\begin{array}{c}\text { Correlation coefficients (r) } \\
\text { Preoperative EQ-5D }\end{array}$ \\
\hline WOMAC-Pain & $0.62^{* *}$ & $-0.60^{* *}$ \\
6MWT (m) & $-0.46^{* *}$ & $0.46^{* *}$ \\
TUG (sec) & $0.46^{* *}$ & $-0.38^{* *}$ \\
Gait Speed (m/sec) & 0.10 & 0.19 \\
Cadence (steps/min) & 0.07 & 0.13 \\
Stride length (cm) & 0.81 & 0.08 \\
Gait cycle duration (sec) & -0.09 & -0.16 \\
Stance phase duration (\% of gait cycle) & 0.20 & -0.11 \\
Swing phase duration (\% of gait cycle) & -0.21 & -0.11 \\
Double support duration (\% of gait cycle) & 0.18 & -0.13 \\
Single support duration (\% of gait cycle) & -0.21 & 0.12 \\
PT extensor of surgical knee (Nm) & -0.25 & 0.15 \\
PT extensor of non-surgical knee (Nm) & $-0.27^{*}$ & 0.13 \\
PT flexor of surgical knee (Nm) & -0.22 & 0.12 \\
PT flexor of non-surgical knee (Nm) & $-0.32^{*}$ & 0.20 \\
H/Q ratio of surgical knee (\%) & 0.16 & -0.18 \\
H/Q ratio of non-surgical knee (\%) & 0.09 & -0.10 \\
Deficit of extensor (\%) & 0.06 & -0.12 \\
Deficit of flexor $(\%)$ & -0.17 & 0.06 \\
\hline Values repesent & & \\
\hline
\end{tabular}

Values represent correlation coefficients (r)

$* \mathrm{p}<0.05, * * \mathrm{p}<0.1$

Table 4. Factors predictive of self-reported physical function and quality of life by multi variat linear regression analysis

\begin{tabular}{lccc}
\hline Outcome/Independent predictor & Standardized $\beta$ & $\mathrm{p}$-value & Adjusted $\mathrm{R}^{2}$ \\
\hline Preoperative WOMAC-Function & & & \\
WOMAC-Pain & 0.55 & $<0.001^{*}$ & 0.49 \\
TUG & 0.34 & $0.002^{*}$ & \\
Preoperative EQ-5D & & & \\
WOMAC-Pain & -0.51 & $<0.001^{*}$ & 0.45 \\
6MWT & 0.30 & $0.008^{*}$ & \\
\hline$* \mathrm{p}<0.05$ & & &
\end{tabular}

shown that the perceived self-reported pain 17, functional outcomes 18, and walking ability 19 of patients who are scheduled to undergo TK are significant predictors of their self-reported physical function. It should be noted that our study was significantly more exhaustive than these three studies, which only examined the correlations listed above: our study assessed how both self-reported physical function and quality of life correlated with multiple physical test variables.

As expected, of all the variables that we measured, preoperative pain associated most strongly with both self-reported physical function and quality of life. This reflects the fact that knee OA is characterized by a progressive decline in strength and flexibility that associates with increased knee joint pain ${ }^{28)}$. The pain, along with the reduction in functional ability, ultimately promotes the decision to undergo TKA because this procedure is known to significantly improve both the pain and function of patients with end-stage arthritis of the knee ${ }^{16,29)}$.

The second variable that correlated most strongly with both perceived physical function and quality of life in our study was dynamic balance, as measured by the TUG test. This is particularly significant because the TUG test involves complex functional movements that are performed every day, namely, standing up from a seated position in a chair, walking, and turning while walking. This may explain why the linear regression analysis showed that the TUG test, but not the 6MWT, was the second most significant independent predictor of self-reported physical function: unlike the 6MWT, the TUG tests a combination of walking ability, lower limb strength, and balance, and therefore its value more closely reflects the impairment of the daily living activities in end-stage knee OA that is being measured by the WOMAC-Function questionnaire. Thus, 
a decline in dynamic balance strongly causes patients with end-stage knee OA to feel that their daily living functions and quality of life have worsened.

The 6MWT measures the maximal distance a subject can walk in 6 minutes and thus is a simple clinically relevant measure of exercise tolerance. Several studies have found modest relationships between measured ambulation and selfreported function in many different patient groups, including patients who are due to undergo TKA ${ }^{30-34)}$. Moreover, it is a moderately valid indicator of submaximal ${ }^{35)}$ and maximal aerobic capacity in patients with knee $\mathrm{OA}^{36)}$. The study showed that preoperative exercise tolerance and walking capacity, as measured by the $6 \mathrm{MWT}$, associated significantly with both the self-reported physical function and quality of life of our patients. This is important because it suggests that aerobic exercise that enhances exercise tolerance may improve the pre- and postoperative functional status and quality of life of patients who are scheduled to undergo TKA.

Interestingly, the extensor and flexor strength of the nonsurgical knee also correlated significantly with self-reported physical function. This probably reflects the fact that the development and progression of joint degeneration associate strongly with loss of muscle strength ${ }^{37,38)}$, and that loss of muscle strength is the strongest single predictor of functional limitations in patients with knee $\mathrm{OA}^{39}$ ). Since patients with knee OA are likely to rely on the more intact (nonsurgical) knee during daily life, weakness of this knee may heighten the perception of these patients that their physical function has become compromised. This notion is supported by a study that showed that the strength of the nonsurgical knee plays an important role in the ability of patients to complete performance-based functional assessments ${ }^{40)}$. One of our main study objectives was to identify potential preoperative rehabilitation interventions that minimize the residual postoperative disability after TKA. Our study shows that preoperative rehabilitation interventions that aim to improve dynamic balance, exercise tolerance, and knee extensor and flexor strength could reduce residual disability after TKA.

This study has several limitations. First, it may not be possible to generalize our results to all patients with end-stage knee OA who are scheduled to undergo TKA. For instance, there are only the data of the patients with knee OA who were due to undergo their first TKA in this study. It remains unclear whether the relationships between perceived physical function and quality of life and physical test variables will also be observed in knee OA patients who are scheduled to receive revision TKA or in patients who are to undergo a first TKA for a non-OA diagnosis. Second, only the preoperative data were analyzed. This meant that the study could not show how TKA changed perceived physical function and quality of life, and how these changes correlated with physical test variables over time. However, the study is continuing as the cohort study and thus will be able to address these questions in due course. Finally, the sample size in this study was relatively small. Thus, future analyses may examine if it is possible to insert a larger proportion of variance in predicting physical function using these variables.

In conclusion, this study showed that South Korean patients with end-stage knee OA who were awaiting a TKA had a profoundly reduced preoperative physical function. Moreover, the perceived physical function of these patients was found to be strongly predicted by the degree of preoperative pain and dynamic balance. In addition, preoperative pain and exercise tolerance were the most powerful predictors of quality of life in these patients. Since functional capacity after TKA strongly associates with preoperative functional capacity, our observations suggest that preoperative rehabilitation strategies that focus on dynamic balance, aerobic, and resistance exercises may be particularly important for these patients, and that preoperative pain, dynamic balance, and exercise tolerance (all of which are readily measured variables) should be routinely assessed when determining the preoperative rehabilitation needs of the TKA candidate.

\section{ACKNOWLEDGEMENT}

This work was supported by the Academic Research Foundation of Jeju National Uninersity Institute of Medical Science in 2014.

\section{REFERENCES}

1) Guccione AA, Felson DT, Anderson JJ, et al.: The effects of specific medical conditions on the functional limitations of elders in the Framingham Study. Am J Public Health, 1994, 84: 351-358. [Medline] [CrossRef]

2) Peat G, McCarney R, Croft P: Knee pain and osteoarthritis in older adults: a review of community burden and current use of primary health care. Ann Rheum Dis, 2001, 60: 91-97. [Medline] [CrossRef]

3) Oliveria SA, Felson DT, Reed JI, et al.: Incidence of symptomatic hand, hip, and knee osteoarthritis among patients in a health maintenance organization. Arthritis Rheum, 1995, 38: 1134-1141. [Medline] [CrossRef]

4) Kennedy LG, Newman JH, Ackroyd CE, et al.: When should we do knee replacements? Knee, 2003, 10: 161-166. [Medline] [CrossRef]

5) Jones CA, Voaklander DC, Suarez-Alma ME: Determinants of function after total knee arthroplasty. Phys Ther, 2003, 83: 696-706. [Medline]

6) Fortin PR, Penrod JR, Clarke AE, et al.: Timing of total joint replacement affects clinical outcomes among patients with osteoarthritis of the hip or knee. Arthritis Rheum, 2002, 46: 3327-3330. [Medline] [CrossRef]

7) Hinman RS, Bennell KL, Metcalf BR, et al.: Balance impairments in individuals with symptomatic knee osteoarthritis: a comparison with matched controls using clinical tests. Rheumatology (Oxford), 2002, 41: 1388-1394. [Medline] [CrossRef]

8) Coudeyre E, Jardin C, Givron P, et al.: Could preoperative rehabilitation modify postoperative outcomes after total hip and knee arthroplasty? Elaboration of 
French Clinical Practice Guidelines, 2007, 50: 189-197.

9) Callahan CM, Drake BG, Heck DA, et al.: Patient outcomes following tricompartmental total knee replacement. A meta-analysis. JAMA, 1994, 271: 13491357. [Medline] [CrossRef]

10) Hawker GA, Wright JG, Coyte PC, et al.: Determining the need for hip and knee arthroplasty: the role of clinical severity and patients' preferences. Med Care, 2001, 39: 206-216. [Medline] [CrossRef]

11) Hawker GA, Guan J, Croxford R, et al.: A prospective population-based study of the predictors of undergoing total joint arthroplasty. Arthritis Rheum, 2006, 54: 3212-3220. [Medline] [CrossRef]

12) Wittink H, Rogers W, Sukiennik A, et al.: Physical functioning: self-report and performance measures are related but distinct. Spine, 2003 , 28 : $2407-2413$. [Medline] [CrossRef]

13) Hidding A, van Santen M, De Klerk E, et al.: Comparison between self-report measures and clinical observations of functional disability in ankylosing spondylitis, rheumatoid arthritis and fibromyalgia. J Rheumatol, 1994, 21: 818-823. [Medline]

14) Fitzgerald GK, Piva SR, Irrgang JJ, et al.: Quadriceps activation failure as a moderator of the relationship between quadriceps strength and physical function in individuals with knee osteoarthritis. Arthritis Rheum, 2004, 51: 40-48. [Medline] [CrossRef]

15) Stratford PW, Kennedy D, Pagura SM, et al.: The relationship between self-report and performance-related measures: questioning the content validity of timed tests. Arthritis Rheum, 2003, 49: 535-540. [Medline] [CrossRef]

16) Riddle DL, Wade JB, Jiranek WA, et al.: Preoperative pain catastrophizing predicts pain outcome after knee arthroplasty. Clin Orthop Relat Res, 2010, 468: 798-806. [Medline] [CrossRef]

17) Rossi MD, Hasson S, Kohia M, et al.: Mobility and perceived function after total knee arthroplasty. J Arthroplasty, 2006, 21: 6-12. [Medline] [CrossRef]

18) Ko V, Naylor JM, Harris IA, et al.: The six-minute walk test is an excellent predictor of functional ambulation after total knee arthroplasty. BMC Musculoskelet Disord, 2013, 14: 145. [Medline] [CrossRef]

19) Bellamy N: Validation study of WOMAC: a health status instrument for measuring clinically-important patient-relevant outcomes following total hip or knee arthroplasty in osteoarthritis. J Orthop Rheumatol, 1988, 1: 95-108.

20) Kim MH, Cho YS, Uhm WS, et al.: Cross-cultural adaptation and validation of the Korean version of the EQ-5D in patients with rheumatic diseases. Qual Life Res, 2005, 14: 1401-1406. [Medline] [CrossRef]

21) Cattaneo D, Regola A, Meotti M: Validity of six balance disorders scales in persons with multiple sclerosis. Disabil Rehabil, 2006, 28: 789-795. [Medline] [CrossRef]

22) Podsiadlo D, Richardson S: The timed “Up \& Go": a test of basic functional mobility for frail elderly persons. J Am Geriatr Soc, 1991, 39: 142-148. [Medline] [CrossRef]

23) Bugané F, Benedetti MG, Casadio G, et al.: Estimation of spatial-temporal gait parameters in level walking based on a single accelerometer: validation on normal subjects by standard gait analysis. Comput Methods Programs Biomed, 2012, 108: 129-137. [Medline] [CrossRef]

24) Maffiuletti NA, Bizzini M, Desbrosses K, et al.: Reliability of knee extension and flexion measurements using the Con-Trex isokinetic dynamometer. Clin Physiol Funct Imaging, 2007, 27: 346-353. [Medline] [CrossRef]

25) Thorstensson A, Karlsson J: Fatiguability and fibre composition of human skeletal muscle. Acta Physiol Scand, 1976, 98: 318-322. [Medline] [CrossRef]

26) Kahn TL, Soheili AC, Schwarzkopf R: Poor WOMAC scores in contralateral knee negatively impact TKA outcomes: data from the osteoarthritis initiative. J Arthroplasty, 2014, 29: 1580-1585. [Medline] [CrossRef]

27) van Leeuwen DM, de Ruiter CJ, Nolte PA, et al.: Preoperative strength training for elderly patients awaiting total knee arthroplasty. Rehabil Res Pract, 2014, 2014: 462750. [Medline]

28) Creamer P: Current perspectives on the clinical presentation of joint pain in human OA. Novartis Found Symp, 2004, 260: 64-78.

29) Lingard EA, Katz JN, Wright EA, et al. Kinemax Outcomes Group: Predicting the outcome of total knee arthroplasty. J Bone Joint Surg Am, 2004, 86-A: 2179-2186. [Medline]

30) Bade MJ, Kohrt WM, Stevens-Lapsley JE: Outcomes before and after total knee arthroplasty compared to healthy adults. J Orthop Sports Phys Ther, 2010, 40: 559-567. [Medline] [CrossRef]

31) de Groot IB, Bussmann HJ, Stam HJ, et al.: Small increase of actual physical activity 6 months after total hip or knee arthroplasty. Clin Orthop Relat Res, 2008, 466: 2201-2208. [Medline] [CrossRef]

32) Mizner RL, Petterson SC, Clements KE, et al.: Measuring functional improvement after total knee arthroplasty requires both performance-based and patientreport assessments: a longitudinal analysis of outcomes. J Arthroplasty, 2011, 26: 728-737. [Medline] [CrossRef]

33) Moffet H, Collet JP, Shapiro SH, et al.: Effectiveness of intensive rehabilitation on functional ability and quality of life after first total knee arthroplasty: a single-blind randomized controlled trial. Arch Phys Med Rehabil, 2004, 85: 546-556. [Medline] [CrossRef]

34) Petterson SC, Mizner RL, Stevens JE, et al.: Improved function from progressive strengthening interventions after total knee arthroplasty: a randomized clinical trial with an imbedded prospective cohort. Arthritis Rheum, 2009, 61: 174-183. [Medline] [CrossRef]

35) Stillwell K, Forman D, McElwain D, et al.: The 6 minute walk test for evaluation of functional capacity in elderly adults 907. Med Sci Sports Exerc, 1996, 28: 152. [CrossRef]

36) Rejeski WJ, Ettinger WH Jr, Schumaker S, et al.: Assessing performance-related disability in patients with knee osteoarthritis. Osteoarthritis Cartilage, 1995, 3: 157-167. [Medline] [CrossRef]

37) Slemenda C, Heilman DK, Brandt KD, et al.: Reduced quadriceps strength relative to body weight: a risk factor for knee osteoarthritis in women? Arthritis Rheum, 1998, 41: 1951-1959. [Medline] [CrossRef]

38) Brandt KD, Heilman DK, Slemenda C, et al.: Quadriceps strength in women with radiographically progressive osteoarthritis of the knee and those with stable radiographic changes. J Rheumatol, 1999, 26: 2431-2437. [Medline]

39) McAlindon TE, Cooper C, Kirwan JR, et al.: Determinants of disability in osteoarthritis of the knee. Ann Rheum Dis, 1993, 52: 258-262. [Medline] [CrossRef]

40) Mizner RL, Petterson SC, Snyder-Mackler L: Quadriceps strength and the time course of functional recovery after total knee arthroplasty. J Orthop Sports Phys Ther, 2005, 35: 424-436. [Medline] [CrossRef] 